\title{
Tratamento endovascular das fístulas carótido-cavernosas
}

\author{
Sávio Boechat Primo de Siqueira1, Carlos Maurício Primo de Siqueira², \\ José Alberto Landeiro ${ }^{3}$, Orlando Maia Junior ${ }^{2-4}$
}

Serviço de Neurocirurgia do Hospital de Força Aérea do Galeão, Rio de Janeiro, RJ, Brasil

\section{RESUMO}

Objetivo: Apresentar o resultado do tratamento endovascular das fístulas carótido-cavernosas. Método: Os autores apresentam sete casos de fístula carótido-cavernosa, dois do tipo dural e cinco casos de fístula direta. Todos os casos apresentavam-se sintomáticos na época do diagnóstico e foram confirmados por arteriografia digital cerebral dos quatro vasos, e todos tratados exclusivamente pelo método endovascular. Resultado: Em todos os casos houve importante melhora dos sintomas clínicos e não houve complicações operatórias ou tardias. Em um paciente foram necessárias duas intervenções para oclusão completa da fístula. Conclusão: O método revelou-se seguro e eficaz. Esta técnica, atualmente, é a primeira opção de tratamento das fístulas carótido-cavernosas em nosso Serviço.

\section{PALAVRAS-CHAVE}

Fístula carótido-cavernosa. Seio cavernoso. Tratamento endovascular.

\section{ABSTRACT}

Carotid-cavernous fistula. Endovascular treatment

Objective: To present and discuss the results of endovascular treatment of carotid cavernous fistula. Method: The authors present seven cases of carotid-cavernous fistulae divided into two groups: dural fistulas and direct carotid-cavernous fistulas. All patients were symptomatic at the time of diagnosis; the definitive diagnosis was confirmed by cerebral angiography. All cases were treated by endovascular procedures. Results: All patients had the fistula occluded; one needed a two stage embolization to achieve total occlusion; there were no complications related to the procedure; preoperative symptoms improved in all. Conclusion: The endovascular procedure showed to be effective and safe and is the first therapeutic choice for carotid cavernous fistula in our Service.

\section{KEY WORDS}

Carotid cavernous fistula. Cavernous sinus. Endovascular treatment.

\section{Introdução}

O seio cavernoso é alvo de muito estudo e fascínio por parte da comunidade científica. Teve sua primeira descrição por Ridley, em $1732^{19}$, "como um seio circular", um espaço venoso perisselar ${ }^{19}$. Neste mesmo ano, após estudo em cadáveres, Winslow ${ }^{19}$ descreveu a presença de trabéculas dentro deste seio venoso, ganhando a nômina anatômica de seio cavernoso ${ }^{19}$. Em 1964, Dwight Parkinson descreveu em detalhes a anatomia microcirúrgica do seio cavernoso, dando um grande passo na abordagem cirúrgica das fístulas carótido-cavernosas, porém, com resultados não muito animadores ${ }^{1,19}$.

As fístulas carótido-cavernosas (FCC), raras na prática neurocirúrgica, consistem em comunicações anormais entre a artéria carótida interna e seus ramos com o seio cavernoso e apresentam o trauma como fator causal mais freqüente ${ }^{7,11,13,15,17}$. Foram feitas diversas classificações para as FCC, a mais aceita foi descrita por Barrow, baseada nas comunicações entre a artéria carótida e seus ramos e o seio cavernoso ${ }^{2,12}$. Essa classificação reconhece quatro tipos de fístulas:

1 Médico residente do Serviço de Neurocirurgia do Hospital de Força Aérea do Galeão (HFAG).

2 Chefe do Serviço de Neurocirurgia do Hospital São José do Avaí.

3 Chefe do Serviço de Neurocirurgia do HFAG, MD, PhD.

4 Neurocirurgião endovascular do Serviço de Neurocirurgia do HFAG. 
1. Tipo A (direta): a artéria carótida interna comunica-se diretamente com o seio cavernoso;

2. Tipo B (indireta): a artéria carótida interna comunica-se com o seio cavernoso por ramos durais;

3. Tipo $\mathrm{C}$ (indireta): a artéria carótida externa comunica-se com o seio cavernoso por ramos durais;

4. Tipo D (indireta): as artérias carótida interna e externa comunicam-se com o seio cavernoso por ramos durais.

As FCC diretas apresentam fisiopatologia diferente das fístulas durais. As diretas são mais comuns, podem ser secundárias a traumas - principalmente quando relacionadas à fratura de base do crânio -, ou espontâneas após a ruptura de aneurisma da porção cavernosa da carótida. O tamanho da laceração da fístula direta é proporcionalmente maior, quando comparada com a fístula dural, particularizando a diferença do tratamento endovascular entre elas ${ }^{20}$.

Clinicamente, as fístulas diretas e indiretas manifestam-se de forma semelhante.O diagnóstico é baseado na história clínica, exame físico e exames radiológicos. O método diagnóstico complementar, padrão-ouro, é a arteriografia digital seletiva da artéria carótida, que evidencia o tamanho e estima o débito da fístula. Têm papel adjuvante no diagnóstico a ressonância magnética, angio-tomografia computadorizada, saturação de bulbo de jugular e Doppler transcraniano ${ }^{3,5,7,8,21}$.

O objetivo deste trabalho é descrever o emprego da técnica endovascular no tratamento das fístulas carótido-cavernosas diretas e indiretas, em sete pacientes, com ilustração de dois casos, e revisão da literatura.

\section{Casuística e método}

Foram selecionados sete pacientes portadores de FCC, através de um estudo retrospectivo, no período de julho de 2003 a julho de 2005, em dois centros de referência da região - Hospital de Força Aérea do Galeão e Hospital São José do Avaí.

Todos os pacientes foram tratados por via endovascular. Foram cinco casos de fístula tipo A ou direta e dois casos de fístula dural indireta (um do tipo B e outro do tipo D). Dos cinco pacientes portadores da fístula direta, três eram mulheres e dois homens, e a média de idade foi de 41 anos. Os dois pacientes portadores de fístula dural eram homens, com média de idade de 51 anos. Os sete pacientes apresentavam alterações clínicas compatíveis com FCC, o diagnóstico confirmado através da arteriografia digital e eleitos para o tratamento endovascular. No grupo de pacientes portadores de fístula direta, o tratamento proposto foi oclusão da FCC com utilização de balão ou balão e coil.

Em três pacientes utilizou-se apenas balão com oclusão da fístula e nos outros dois casos foram necessários o balão e coils.

Nos dois pacientes portadores de fístula dural foi feita a oclusão do seio cavernoso com a utilização de líquidos adesivos Onix ${ }^{\circledR}$ e Hystoacryl ${ }^{\circledR}$, com cateterização do seio cavernoso através da artéria femoral em um e da veia oftálmica superior no outro.

Todos os pacientes, ao final do procedimento foram submetidos à arteriografia digital de controle. Em um paciente foi visualizada oclusão parcial da fístula direta (caso 1), sendo necessário novo tratamento em segundo tempo, conseguindo-se oclusão total da fístula com uso de coils eletrodestacáveis.

Imediatamente, após os procedimentos, todos os pacientes apresentaram regressão dos sintomas, como proptose, quemose, sopro, tinitus e mantiveram algum grau de paresia de nervos cranianos já existentes (Tabela 1).

\section{Casos ilustrativos}

Caso 5 - JGN, 21 anos de idade, portador de grande FCC direita, associada à acidente automobilístico, descoberta por ocasião de investigação neurorradiológica motivada pelo quadro de proptose, sopro, quemose à direita, paralisia de VI nervo, associado à cefaléia e tinido. Realizou tomografia computadorizada (TC) de crânio e angio-TC que evidenciaram a presença da fístula carótido-cavernosa (Figuras 1 e 2). O estudo angiográfico, revelou a presença de FCC direita, do tipo direta e de grande fluxo. Através do sistema co-axial utilizando-se de microcateter e microguia, depositamos um balão destacável junto ao colo da fístula, obtendo boa oclusão da mesma (Figura 3). O procedimento foi realizado com heparinização plena e anestesia local, sem intercorrências.

No pós-operatório, o paciente evoluiu com melhora da cefaléia, proptose, quemose e do tinido. Manteve discreta paresia do VI nervo.

Caso 7 - PRGP, 40 anos de idade, portador de fistula artério-venosa dural, tipo D (Figura 4), descoberta por ocasião de investigação neurorradiológica motivada por cefaléia, quemose, proptose, plegia de VI, IV e III nervos cranianos à direita (Figura 5).

$\mathrm{O}$ estudo angiográfico revelou a presença de fistula artério-venosa do tipo dural, proveniente dos ramos externos da artéria carótida e múltiplos ramos do sifão carotídeo intracraniano (tipo D), com drenagem por 


\begin{tabular}{|c|c|c|c|c|c|c|c|}
\hline \multicolumn{8}{|c|}{ Tabela 1} \\
\hline & & & & Resumo do & casuística & & \\
\hline Identificação & Caso & $\begin{array}{l}\text { Tipo de } \\
\text { fístula }\end{array}$ & Sintomatologia & Via de acesso & $\begin{array}{c}\text { Tratamento } \\
\text { proposto }\end{array}$ & $\begin{array}{c}\text { Controle } \\
\text { angiográfico final }\end{array}$ & Pós-operatório \\
\hline $\begin{array}{l}\text { EMP } \\
20 \text { anos } \\
18.09 .2004\end{array}$ & 1 & A & $\begin{array}{l}\text { Olho vermelho e } \\
\text { massa pulsátil à } \\
\text { direita, cefaléia }\end{array}$ & Artéria femoral & $\begin{array}{l}\text { Oclusão da fístula } \\
\text { com micromolas }\end{array}$ & $\begin{array}{l}\text { Oclusão parcial da } \\
\text { fístula com visualização } \\
\text { de estase venosa }\end{array}$ & $\begin{array}{l}\text { Melhora da quemose e } \\
\text { diminuição do exoftalmos }\end{array}$ \\
\hline $\begin{array}{l}\text { EMP } \\
21 \text { anos } \\
28.01 .2005\end{array}$ & 1 & A & $\begin{array}{l}\text { Pequena } \\
\text { exoftalmia }\end{array}$ & Artéria femoral & $\begin{array}{l}\text { Oclusão da fístula } \\
\text { com micromolas e } \\
\text { balão destacável }\end{array}$ & Ausência de fístula & $\begin{array}{l}\text { Desaparecimento da } \\
\text { exoftalmia }\end{array}$ \\
\hline $\begin{array}{l}\text { FNC } \\
63 \text { anos } \\
25.03 .2005\end{array}$ & 2 & B & $\begin{array}{l}\text { Quemose e } \\
\text { proptose bilateral }\end{array}$ & $\begin{array}{l}\text { Via direta pela } \\
\text { veia oftálmica } \\
\text { superior }\end{array}$ & Histoacryl & $\begin{array}{l}\text { Ausência de fístula } \\
\text { residual }\end{array}$ & Melhora dos sintomas \\
\hline $\begin{array}{l}\text { MCR } \\
59 \text { anos } \\
15.10 .2004\end{array}$ & 3 & A & $\begin{array}{l}\text { Sopro, quemose, } \\
\text { proptose com } \\
\text { paresia de III, } \\
\text { IV e VI nervos à } \\
\text { direita }\end{array}$ & Artéria femoral & $\begin{array}{l}\text { Micromolas e } \\
\text { balão }\end{array}$ & $\begin{array}{l}\text { Oclusão do vaso } \\
\text { portador (Carótida dir.) }\end{array}$ & $\begin{array}{l}\text { Paciente sem novos déficits. } \\
\text { Manteve paresia de nervos } \\
\text { cranianos }\end{array}$ \\
\hline $\begin{array}{l}\text { MM } \\
69 \text { anos } \\
17.07 .2004\end{array}$ & 4 & A & $\begin{array}{l}\text { Sopro, proptose, } \\
\text { quemose, à esq.; } \\
\text { hemorragia. em } \\
\text { chama de vela }\end{array}$ & Artéria femoral & $\begin{array}{l}\text { Balão } \\
\text { destacável }\end{array}$ & $\begin{array}{l}\text { Oclusão total da } \\
\text { fístula. Ausência } \\
\text { de fenômenos } \\
\text { tromboembólicos }\end{array}$ & Melhora dos sintomas \\
\hline $\begin{array}{l}\text { JGN } \\
21 \text { anos } \\
19.07 .2005\end{array}$ & 5 & A & $\begin{array}{l}\text { Sopro, proptose, } \\
\text { quemose, e } \\
\text { paresia de VI } \\
\text { nervo à direita }\end{array}$ & Artéria femoral & $\begin{array}{l}\text { Balão } \\
\text { destacável }\end{array}$ & Oclusão da fístula & $\begin{array}{l}\text { Paciente sem novos déficits. } \\
\text { Melhora dos sintomas, } \\
\text { manteve paresia de VI nervo }\end{array}$ \\
\hline $\begin{array}{l}\text { JCCS } \\
36 \text { anos } \\
\text { associado à fístula } \\
\text { vértebro-vertebral } \\
\text { esq. } \\
18.05 .2005\end{array}$ & 6 & A & $\begin{array}{l}\text { Sopro, proptose, } \\
\text { quemose e tinido } \\
\text { a dir }\end{array}$ & Artéria femoral & $\begin{array}{l}\text { Balão destacável } \\
\text { na FCC e stent } \\
\text { recoberto na fístula } \\
\text { vértebro-vertebral }\end{array}$ & $\begin{array}{l}\text { Oclusão da fístula } \\
\text { carótido-cavernosa e } \\
\text { vértebro-vertebral }\end{array}$ & $\begin{array}{l}\text { Ausência da fístula, bom } \\
\text { posicionamento do stent }\end{array}$ \\
\hline $\begin{array}{l}\text { PRGP } \\
40 \text { anos } \\
08 / 11 / 2004\end{array}$ & 7 & $\mathrm{D}$ & $\begin{array}{l}\text { Cefaléia, } \\
\text { proptose, } \\
\text { quemose, à dir. } \\
\text { com paresia dos } \\
\text { nervos cranianos: } \\
\text { III, IV, VI à dir. }\end{array}$ & $\begin{array}{l}\text { Via artéria } \\
\text { femoral, acesso à } \\
\text { fístula através da } \\
\text { artéria meníngea } \\
\text { média }\end{array}$ & Hystoacril & $\begin{array}{l}\text { Oclusão da fístula. } \\
\text { Com ausência de } \\
\text { fístula residual } \\
\text { e fenômenos } \\
\text { tromboembólicos }\end{array}$ & $\begin{array}{l}\text { Melhora clínica } \\
\text { imediata. Manteve } \\
\text { discreta ptose palpebral } \\
\text { direita }\end{array}$ \\
\hline
\end{tabular}

inúmeras veias do córtex cerebral em direção ao seio cavernoso homolateral (Figura 4).

O procedimento foi realizado sob anestesia geral, com heparinização plena e cateterização, via artéria femoral. Através do sistema co-axial, utilizamos micro cateter ultra flow 1.5 e micro guia SL 0.10 , cateterizamos seletivamente a fístula através de um ramo da artéria carótida extena direita e depositamos em seu interior uma mistura contendo cola $\left(\right.$ Hystoacril $^{\circledR}$ ) (Figura 6). Os controles angiográficos mostraram boa deposição do material emboligênico no interior da fistula obstruindo-a totalmente, sem apresentar fenômenos tromboembólicos (Figura 7).
O paciente evoluiu, no pós-operatório imediato, com melhora clínica, permanecendo com discreta ptose palpebral à direita (Figura 8 ).

\section{Discussão}

Com o advento de novas técnicas de neuroimagem e radiologia intervencionista, o neurocirurgião vem aprofundando seus conhecimentos nas doenças cérebro- 


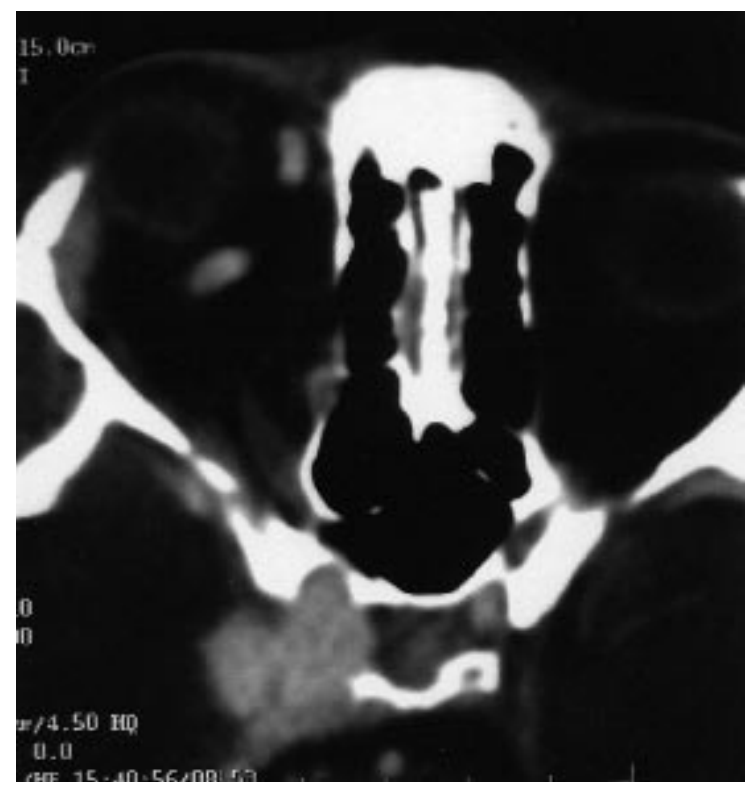

Figura 1 - Caso 5. TC de crânio mostrando imagem hiperdensa parasselar em topografia do seio cavernoso.

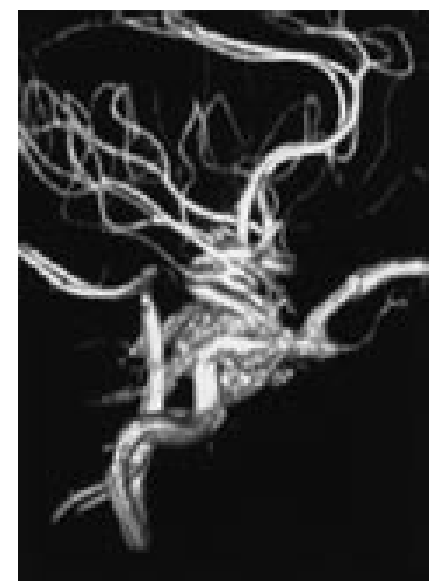

Figura 2 - Caso 5. Angio-TC de crânio evidenciando grande fístula carótido-cavernosa.

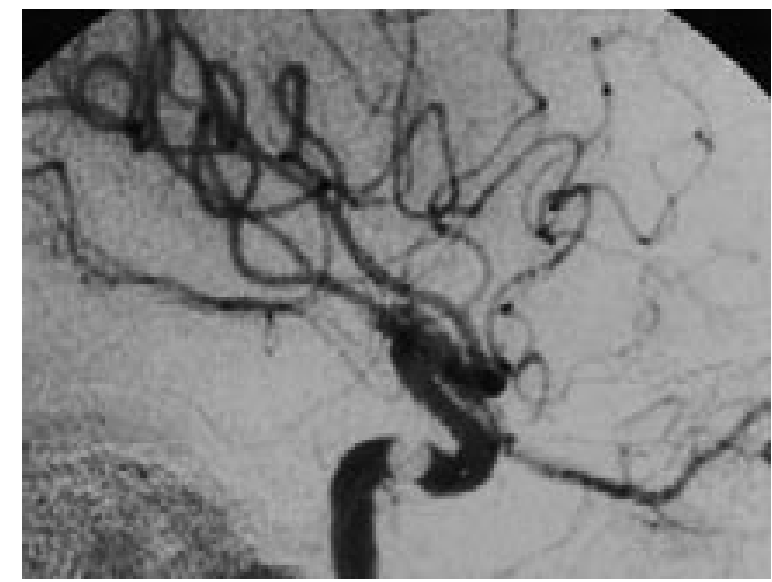

Figura 3 - Caso 5. Controle final com angiografia da artéria carótida direita em perfil, mostrando oclusão total da fístula pelo balão e preservação da artéria.

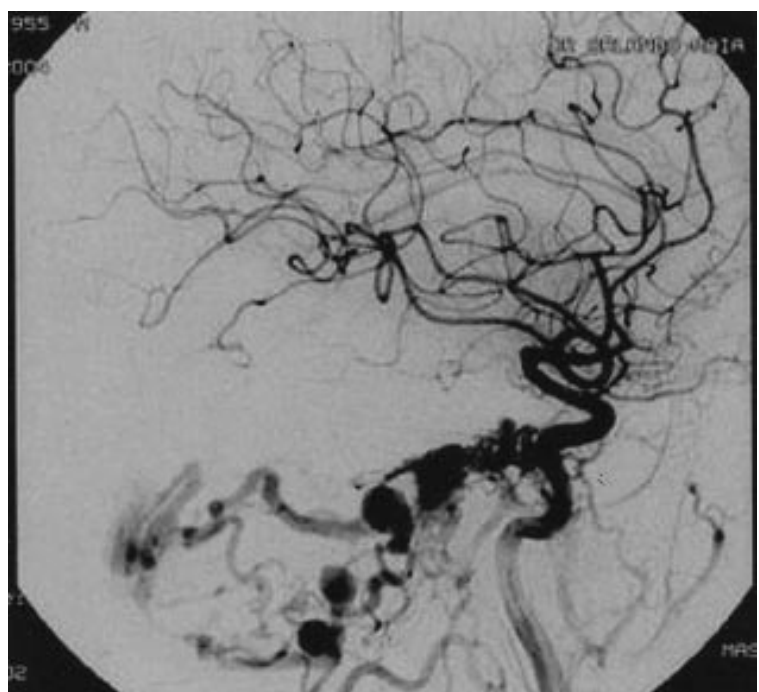

Figura 4 - Caso 7. Fístula dural, tipo D, nutrida por ramos de carótida interna e externa direita.

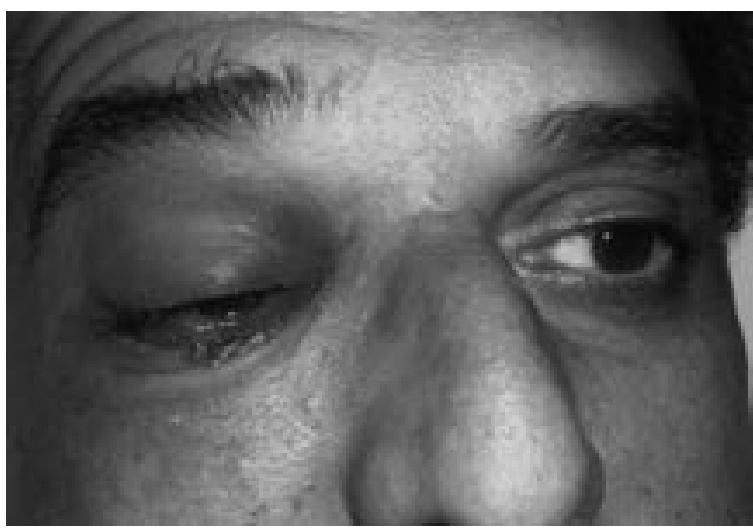

Figura 5 - Caso 7. Proptose, ptose, hiperemia conjuntival e oftalmoplegia completa.

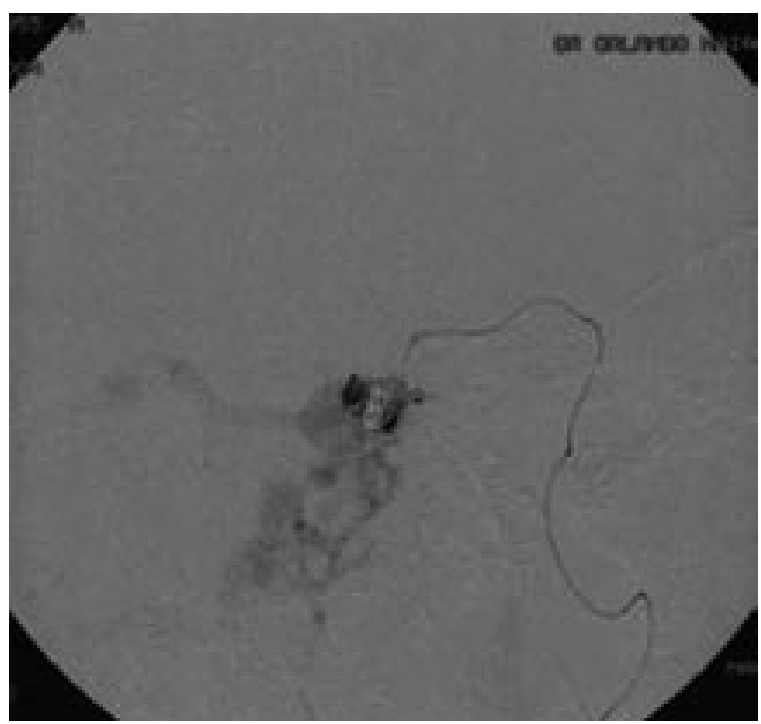

Figura 6 - Caso 7. Microcateter seletivo dentro da fístula, liberando material emboligênico. 


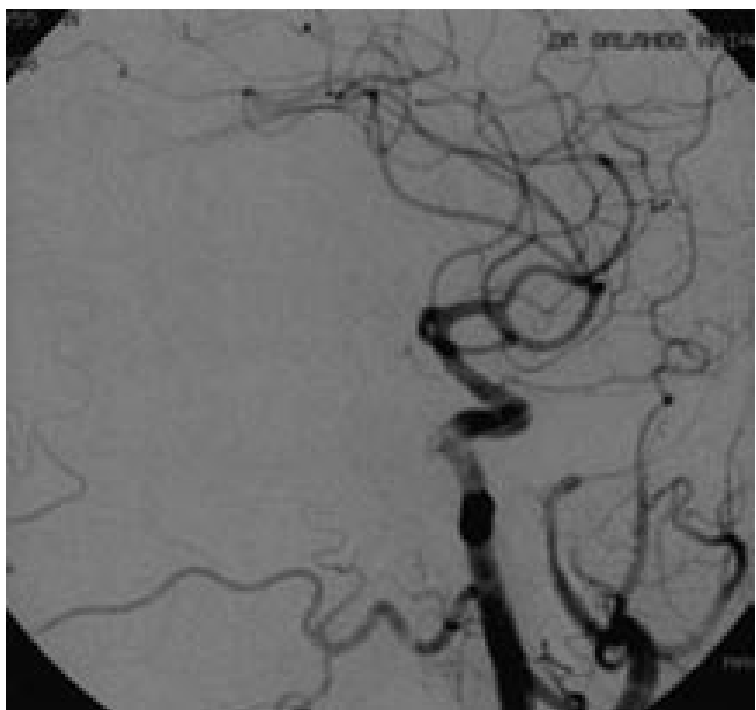

Figura 7 - Caso 7. Angiografia de controle, da artéria carótida direita, mostrando oclusão completa da fístula.

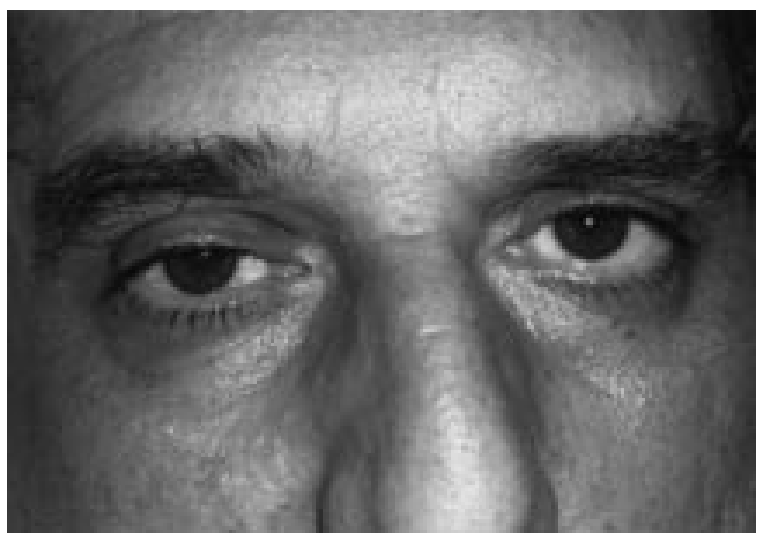

Figura 8 - Caso 7. No pós-operatório, mobilidade ocular preservada, persistindo leve ptose à direita.

vasculares e optando por tratamentos mais eficazes, seguros e menos invasivos das FCC.

Do ponto de vista fisiopatológico, é importante a compreensão das alterações hemodinâmicas que ocorrem nos compartimentos venosos, com especial atenção ao seio cavernoso.

Após o estabelecimento da fístula artério-venosa ocorre transmissão do fluxo e da pressão arterial para o seio cavernoso, provocando dilatação venosa e reversão do fluxo nas veias que drenam para o seio cavernoso, bem como o aumento do fluxo nas suas veias de drenagem habituais (seio petroso inferior e plexo pterigóide $)^{6}$.

As primeiras veias a receberem o fluxo reverso são as veias oftálmicas superiores (VOS) e veias oftálmicas inferiores (VOI), cursando com ingurgitamento, congestão orbitária, aumento da pressão intra-ocular e impedimento de drenagem do humor aquoso, levando a glaucoma secundário ${ }^{6,16,18}$. As pressões venosa e intra-ocular elevadas podem comprometer a perfusão da retina e resultar em diminuição da acuidade visual, uma emergência neurocirúrgica ${ }^{6}$. Edema de estruturas orbitárias decorrente do impedimento da drenagem venosa pode levar a limitações mecânicas da movimentação dos músculos extra-oculares que, por vezes, já têm sua função comprometida pela compressão exercida sobre os nervos cranianos.

Os seios intercavernosos podem transmitir para o outro lado as mesmas alterações hemodinâmicas ocorridas do lado da fístula, levando a quadro clínico semelhante.

O tratamento das FCC sofreu mudanças contínuas ao longo do tempo, impulsionadas pelo insucesso das técnicas utilizadas até o passado recente e pela melhor compreensão da fisiopatologia das $\mathrm{FCC}^{6}$.

Em 1972, Serbinenko desenvolveu o primeiro balão, de uso transarterial, para o tratamento das $\mathrm{FCC}^{22}$. Hoje, os bons resultados obtidos com as técnicas endovasculares na utilização de balões destacáveis, tornaram a embolização o tratamento de escolha nas FCC diretas ${ }^{1,5,9,14}$. O balão permite a oclusão do orifício fistuloso, sem a ligadura do vaso portador (artéria carótida interna), em $80 \%$ dos $\operatorname{casos}^{20}$. Se o tamanho da fístula for muito grande, incompatível com o tamanho do balão, podemos lançar mão de coils e hidrocoils eletro-destacáveis, com intuito de diminuir o tamanho da fístula, permitindo a oclusão da fístula remanescente com balão ${ }^{14}$.

Para o tratamento das FCC indiretas tem sido preconizada a via venosa como eleição, promovendo a cateterização retrógrada pelas veias facial transjugular e oftálmica superior, com intuito de se cateterizar seletivamente o seio cavernoso, liberar em seu interior material emboligênico, produzindo a oclusão do mesmo. Trata-se de via mais segura, com menor potencial artério-trombótico, menor taxa de insucesso terapêutico e de mais fácil realização $0^{5,711,14}$.

Em nossa casuística, dois pacientes (Casos 2 e 7) apresentavam FCC indireta; nestes a fístula foi atingida através dos ramos da artéria carótida externa em um (Caso 7) e, no outro, via direta pela veia oftálmica superior (Caso 2), obtendo-se a oclusão total das fístulas, com utilização de cola e coil. Essa abordagem é limitada para pacientes cujas fístulas apresentem muitas artérias nutrientes, permitindo a oclusão completa das mesmas ${ }^{4}$.

Em todos os casos houve importante melhora dos sintomas clínicos, e não houve complicações operatórias ou tardias. Em um paciente (Caso 1) houve oclusão parcial da fístula no primeiro procedimento sendo necessária segunda intervenção realizada com sucesso. As fístulas diretas foram ocluídas por via arterial e, na 
maioria dos casos, utilizou-se o balão destacável de látex, conforme o relatado na literatura como primeira opção ${ }^{4}$. A via venosa foi utilizada com sucesso em um dos casos de FCC indireta.

\section{Conclusão}

Este trabalho descreveu o emprego do método endovascular no tratamento das FCC diretas e indiretas. Nos sete pacientes apresentados conseguiu-se oclusão total da fístula com remissão dos sinais e sintomas. Não houve complicações e o método revelou-se seguro e eficaz. Esta técnica constitui a primeira opção de tratamento das FCC em nosso Serviço.

\section{Referências}

1. ARAT A, CEKIRGE S, SAATCI I, OZGEN B: Transvenous injection of Onyx for casting of the cavernous sinus for the treatment of a carotid-cavernous fistula. Neuroradiology 46:1012-5, 2004.

2. BARROW DL, SECTOR RH, BRAUN IF, LANDMAN JA, TINDAL SC, TINDAL GT: Classification and treatment of spontaneous carotid cavernous fistula. J Neurosurg 62:24856, 1985.

3. CARRILLO A, VARA F, ABADAL JM, MARSÉ P, IBAÑEZ J, PUEYO J: Jugular venous oxygen monitoring: a helpful technique in the early diagnosis of a traumatic carotid-carvenous sinus fistula. Intens Care Med 24:71-2, 1998.

4. CHENG KM, CHAN CM, CHEUNG YL: Transvenous embolisation of dural carotid-cavernous fistulas by multiple venous routes: a series of 27 cases. Acta Neurochir (Wien) 145:17-29, 2003.

5. DERANG J, YING H, LONG Y et al.: Treatment of carotidocavernous sinus fistulas retrograde via the superior ophthalmic vein (SOV). Surg Neurol 52:286-93, 1999.

6. FRUDIT ME: Fístulas Carótidocavernosas. In: Braga MF, Melo PM (ed): Guias de Medicina Ambulatorial e Hospitalar. UNIFESP/Escola Paulista de Medicina - Neurocirurgia Ed 1. São Paulo, Manole, 2005, cap 42, pp 403-10.

7. KLINK T, HOFMANN E, LIEB W: Trasvenous embolization of carotid cavernous fistulas via the superior ophthalmic vein. Graefe's Arch Clin Exp Opthalmol 239:583-8, 2001.

8. KURATA A, MIYASAKA Y, KUNII $M$ et al.: The value of long-term clinical follow-up for cases of spontaneous carotid cavernous fistula. Acta Neurochir (Wien) 140:65-72, 1998.
9. JANSEN O, DÖRFLER A, FORSTING M, HASTMANN M, VON KUMMER R, TRONNIER V, SARTOR K: Endovascular therapy of arteriovenous fistulae with eletrolytically detachable coils. Neuroradiology 41:951-7, 1999.

10. JU BK, HEE MH, KANG H, CHANG S: Endovascular occlusion of direct carotid cavernous fistula with detachable balloons: usefulness of 3D angiography. Neuroradiology 47:271-81, 2005

11. LIANG CC, MICHON JJ, CHENG KM, CHAN CM, CHEUNG YL: Ophthalmologic outcome of spontaneous carotid-cavernous fistulas: a preliminary report. International Ophthalmology 23:43-7, 1999.

12. LIU HM, HUANG YC, WANG YH, TU YK: Trasarterial embolisation of complex cavernous sinus dural arteriovenous fistulae with low-concentration cynoacrylate. Neuroradiology 42:766-70, 2000.

13. LIU HM, WANG YH, CHEN YF, CHENG JS, YIP PK, TU YK: Long-term clinical outcome of spontaneous carotid cavernous sinus fistulae supplied by dural branches of the internal carotid artery Neuroradiology 43:1007-14, 2001.

14. LO D, VALLEE JN, BITAR A, GUILLEVIN R, LEJEAN L, VAN EFFENTERRE R, CHIRAS J: Endovascular management of carotid-cavernous fistula combined with ipsilateral internal carotid artery occlusion due to gunshot: contra-lateral arterial approach. Acta Neurochir (Wien) 146:403-6, 2004.

15. MEYERS MP, Van HALBACH V, DOWD CF et al.: Am J Ophthalmol 134:85-92, 2002.

16. OHTSUKA K, HASHIMOTO M: Clinical findings in a patient with spontaneous arteriovenous fistulas of the orbit. Am J Ophthalmol 127:736-7, 1999.

17. OSBORN AG: Diagnóstico Neurorradiológico. Rio de Janeiro, Revinter, 1999, pp 284-328.

18. OSBORN AG: Angiografia Cerebral Diagnóstica Ed 2. Rio de Janeiro, Revinter, 2002, pp 277-312.

19. PARKINSON D: A surgical approach to the cavernous portion of the carotid artery. Canad J Surg 12:474-83, 1964.

20. SAWLANI V, PHADKE R, KUMAR S, GUJRAL RB: Guglielmi detachable coils in the treatment of carotid-cavernous fistula. Clin Radiol 59:86-90, 2004.

21. SANJAY G, LARSEN D, DIAZ DJ: Carotid cavernous fistulas. In Youmans JR (ed): Neurological Surgery Ed 5. Philadelphia, Saunders, 2003, vol 2 pp 2341-53.

22. SERBINENKO FA: Ballon catheterization and occlusion of major cerebral vessels. J Nerosurg 54:125-45, 1974.

Original recebido em outubro de 2005

Aceito para publicação em janeiro de 2006

\section{Endereço para correspondência:}

Sávio Boechat Primo de Siqueira

Rua Ministro Otavio Kelly, 457 / ap 402

24220-300-Niterói, RJ

e-mail:sboechatps@yahoo.com.br 